\title{
Correction to: Anti-inflammatory activity of compounds from the rhizome of Cnidium officinale
}

\author{
Huynh Nguyen Khanh Tran ${ }^{1}$ Thao Quyen Cao ${ }^{1} \cdot J^{2}$ ong Ah Kim ${ }^{2} \cdot$ \\ Ui Joung Youn ${ }^{3} \cdot$ Sanghee $\mathrm{Kim}^{3} \cdot$ Mi Hee Woo ${ }^{1} \cdot$ Byung Sun Min ${ }^{1}$
}

Published online: 30 November 2018

(C) The Pharmaceutical Society of Korea 2018

Correction to: Arch. Pharm. Res. (2018) 41:977-985 https://doi.org/10.1007/s12272-018-1048-9
Conflict of interest The authors declare no conflict of interest.

The author would like to include conflict of interest statement of the online published article. The correct conflict of interest statement should read as:

The original article can be found online at https://doi.org/10.1007/s12272-018-1048-9.

Jeong Ah Kim

jkim6923@knu.ac.kr

$\bowtie$ Byung Sun Min

bsmin@cu.ac.kr

1 College of Pharmacy, Drug Research and Development Center, Daegu Catholic University, Gyeongbuk 38430, Republic of Korea

2 College of Pharmacy, Research Institute of Pharmaceutical Sciences, Kyungpook National University, Daegu 41566, Republic of Korea

3 Division of Life Sciences, Korea Polar Research Institute, Incheon 21990, Republic of Korea 